\title{
DESIGUALDADES SOCIOCULTURALES EN EL APRENDIZAJE DE MATEMÁTICA Y LENGUA DE LA EDUCACIÓN SECUNDARIA EN ARGENTINA: UN MODELO DE TRES NIVELES
}

(Sociocultural inequalities in the Learning of Language and Mathematics in Secondary Education in Argentina: A model of three levels)

Article Record
$\underline{\text { About author }}$
$\underline{\text { HTML format }}$

\author{
por \\ Rubén Cervini (racervin@infovia.com.ar)
}

$\underline{\text { Ficha del artículo }}$
$\underline{\text { Sobre el autor }}$
Formato HTML

\begin{abstract}
This article analyzes the effects of family background and the socioeconomic context of the school and state on students' achievements in mathematics and language at the end of secondary education in Argentina. The study examined data of 150,000 students in 3,300 high schools. Multilevel linear modeling with three levels (student, school and state) was used to analyze the family background and context effects on achievement. The results are discussed in term of the theory of distributive justice (Rawls) and the concept of equality of educational opportunity (Coleman). It was found that the educational achievement is affected by the strong socioeconomic segmentation of the institutional educational system and that the cultural capital indicators are the most important predictors.
\end{abstract}

\section{Keywords}

Educational achievement, high school, socioeconomic context, family background, social origins, cultural capital, educational segmentation, Mathematics, Language, equality of educational opportunity, Argentina

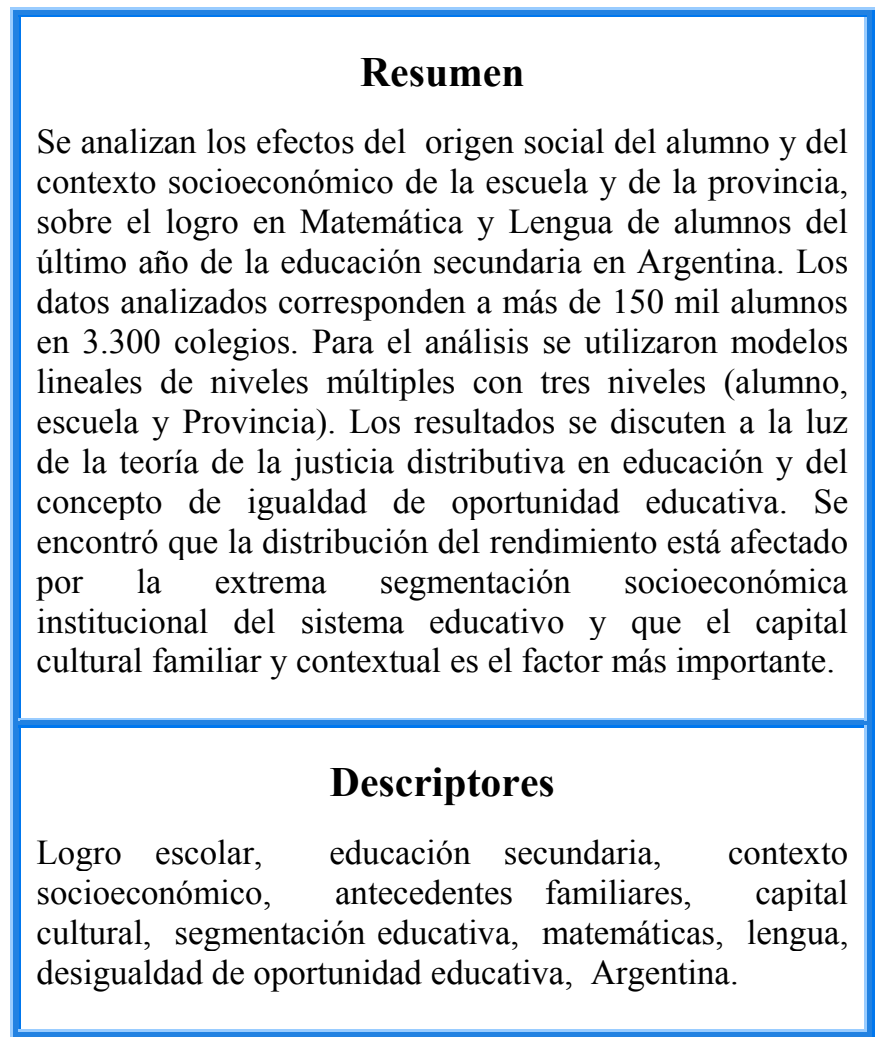




\section{Introducción}

La igualdad de oportunidad educativa ha sido un principio clave del pensamiento democrático y de la justicia solidaria en las sociedades capitalistas. Históricamente, uno de los principales ejes de discusión ha girado en torno de su significado cuando se lo aplica a los 'resultados educativos' (logros de aprendizaje), en contraposición a los 'insumos educativos. En los países desarrollados, razones éticoideológicas y al mismo tiempo instrumentales, han promovido durante décadas, el desarrollo de técnicas de medición de logros educativos y otros conceptos conexos (coeficiente intelectual, aptitud), y de metodologías para el análisis de sus determinantes, dando lugar a una de las más fructíferas tradiciones de investigación empírica. Sus resultados han mostrado, recurrentemente, la existencia de disparidades sociales en los logros educacionales, alimentando continuamente la polémica acerca de cuál es el grado de desigualdad que es razonable considerar como violatorio al principio de igualdad de oportunidades educativas.

En términos generales, la respuesta a ese interrogante varía de acuerdo a cuál sea el nivel educativo de referencia. Cuando el logro se refiere al nivel mínimo (o umbral) de aprendizaje adoptado y considerado por la sociedad como alcanzable $y$ exigible a todos sus miembros, cualquier disparidad significa "desigualdad de oportunidad educativa". En los países desarrollados ese umbral es la educación secundaria, cuya universalización ya ha sido alcanzada. Pero, este no es el caso de los países del Tercer Mundo. La evidencia empírica indica que ese nivel escolar está por encima del umbral educativo universal efectivamente conseguido.

$\mathrm{Si}$ bien Argentina ocupa una posición aventajada en comparación a la mayoría de los países de América Latina, también está lejos de alcanzar la efectiva universalización secundaria [1]. Ello es particularmente notorio cuando se presta atención al egreso de la educación secundaria. Informaciones referidas a 1999 (SIEMPRO,2000) indican que, si bien la cobertura neta secundaria de la población de 13 a 17 años era relativamente alta $-82 \%$-, sólo el $45,7 \%$ de la población de 18 a 24 año había completado la educación secundaria.

En este caso, la respuesta al interrogante inicialmente planteado es diferente: no toda desigualdad de aprendizaje viola el principio de equidad. El camino tradicional para su tratamiento es el análisis de las correlaciones entre el logro académico, por un lado, y determinadas características del estudiante, como por ejemplo, su origen social o su género, por el otro. Tal metodología permite conocer el grado de (in)equidad educativa y simultáneamente, develar el peso relativo que tienen los factores escolares y extra-escolares en la determinación del aprendizaje escolar.

El presente trabajo se ubica en esa perspectiva. $\mathrm{Su}$ principal objetivo es determinar el nivel y la forma de la (in)equidad de los logros educacionales en Matemática y Lengua a la finalización del nivel secundario de Argentina, utilizando como criterios de evaluación el género, el origen social del estudiante (capital económico y capital cultural familiar) y el contexto socioeconómico escolar.

Los datos utilizados para lograr ese objetivo general provienen del Censo Nacional de Finalización del Nivel Secundario de 1998, realizado por el Ministerio de Cultura y Educación de la Nación. Se utilizan los resultados de las pruebas de Matemática y de Lengua y algunas informaciones provenientes del cuestionario aplicado a los estudiantes evaluados. Para el tratamiento de los datos se aplica la técnica estadística "por niveles múltiples" (modelo lineal jerárquico), con tres niveles de agrupamiento - Provincia, colegio y estudiante.

El trabajo se inicia enmarcando el tema de la desigualdad de logros académicos en el enfoque de la teoría distributiva de la justicia (punto I); en el punto II se realiza una breve revisión del tema en la tradición de estudios empíricos en los países desarrollado y del 
Tercer Mundo. A continuación, se expone la definición operacional del concepto de equidad educativa (punto III), con base en el cual se determinan los objetivos específicos y la metodología utilizada (punto IV). En el punto V se exponen los principales resultados, para finalmente, extraer algunas conclusiones (punto VI).

\section{A. Justicia y educación}

La preocupación por la forma de la distribución interindividual de valores socialmente relevantes es propio de las llamadas teorías solidarias de la justicia, en contraposición a la tradición de la filosofía utilitarista, más atenta a la maximización de la suma (o el promedio) de los niveles de bienestar o utilidad de los miembros de la sociedad. La teoría de la justicia distributiva de Rawls (1979) es paradigmática. Asume que, cuando evaluamos cualquier situación social, operan ciertos juicios morales básicos, fundamentos de nuestra intuición moral. Su principal axioma afirma que el hombre tiende a minimizar el máximo perjuicio en una situación de incertidumbre y es ilustrado a través de una situación original, donde las partes involucradas, en un estado "pre-real", deben establecer los principios que regulen la futura "vida real" en sociedad, pero sin saber cuál será el punto de partida social de que gozará cada una de ellas ni cuáles serán sus características personales. Bajo esa situación inicial, que denomina "velo de la ignorancia", y los supuestos de escasez moderada y búsqueda exclusiva del interés propio ("circunstancias de la justicia"), por un lado, y de la distinción entre acción interesada y la moral ("coerción moral"), por el otro, Rawls sustenta que las partes llegarán necesariamente a los siguientes principios: todos tenemos igual derecho (i) "al conjunto más amplio de libertades fundamentales iguales, que sean compatibles con el conjunto de las libertades para todos" (principio de igual libertad) y (ii) a las posibilidades de acceso a las diversas funciones y posiciones (principio de la igualdad equitativa de las posibilidades), que están abiertas a todos y que implican desigualdades económicas y sociales, (iii) sólo aceptables si son "para mayor beneficio de los miembros menos aventajados de la sociedad" (principio de la diferencia).

En esta formulación es posible distinguir resultados (bienes básicos) y posibilidades como objetos de distribución. Los primeros se refieren a diversos tipos de ventajas socioeconómicas ${ }^{[2]}$, concebidas como las condiciones contextuales y los medios generales necesarios para poder alcanzar el bien o estado de bienestar deseado por el individuo (Rawls, 1982:175) [3]. Las posibilidades, en cambio, se refieren tanto a los bienes materiales que recibe un individuo en condiciones de equidad como al acceso a la formación (educación). La sociedad debe asegurar a todos sus miembros la misma posibilidad de alcanzar los requisitos para ocupar las posiciones más aventajadas en la estructura social. Dado que la educación y su certificación aparece como una condición necesaria en la casi totalidad de los casos, es responsabilidad de toda sociedad justa asegurar para todos sus habitantes, a igualdad de talento $y$ capacidades, $y$ deseos de utilizarlos, e independientemente de cualquier otro criterio de discriminación (origen social, género, raza, ubicación geográfica, etc), las mismas posibilidades de acceso a los diferentes niveles de certificación del sistema educativo (Rawls,1979). Es decir, aquellos que poseen un mismo grado de talento y capacidad y además, tienen la misma voluntad de ponerlos en práctica, deberían tener las mismas esperanzas de éxito o de vida, sin importar la ubicación socioeconómica en que hayan nacido.

Así formulada, la propuesta conlleva otra dicotomía: se trata de la igualdad en las posibilidades, pero no en las probabilidades. Las aspiraciones y el decurrente esfuerzo del individuo aparecerían como factores inobjetables. Las desigualdades en la distribución de los logros educacionales debidas a ellos no violarían los principios de la justicia distributiva, o sea, a igualdad en la capacidad y el talento, las desigualdades debidas a la aspiración o al esfuerzo serían legítimas. Entonces, dada la igualdad de 
posibilidades, existen diferentes probabilidades legítimas de acceder a posiciones privilegiadas.

Pero, por otro lado, el propio Rawls piensa que todas las causas que estén más allá del control de la persona son "arbitrarias desde un punto de vista moral" y no podrían legitimar cualquier desigualdad. Entonces, las ventajas proporcionadas por las dotes naturales no serían menos arbitrarias moralmente que las ventajas provenientes de circunstancias sociales. Más aún, se sabe que no solamente las aspiraciones sino también el desarrollo del talento "natural" dependen del medio sociocultural familiar. Por lo tanto, el reclamo de que las perspectivas de vida de aquellos con las mismas capacidades y aspiraciones "no deben ser afectadas por su clase social" no puede soslayar el hecho de que aquellas características son a su vez, afectadas por el origen social. La verdadera igualdad de oportunidades requeriría una igualación notable de las circunstancias familiares. Igualar talento $y$ aspiraciones requeriría tal intervención a nivel familiar que sería casi imposible hacerlo sin violar un principio con mayor prioridad, el de la autonomía familiar (la libertad de educar a los hijos). Por eso, la manera de minimizar el problema, sin herir los principios de libertad, es a través de la disminución del grado de la desigualdad económica y social en la sociedad.

Educación primaria y justicia distributiva. Sin desconocer la imposibilidad fáctica de la perfecta igualdad equitativa de las oportunidades, la dicotomía posibilidad/probabilidad expuesta anteriormente, genera un interrogante clave: ¿Qué debe entenderse por igual posibilidad de acceso cuando el objeto es la educación (principio de la igualdad equitativa de posibilidades)? ¿Existen competencias escolares que deban ser apropiadas por todos los miembros de una sociedad como condición necesaria para alcanzar la igualdad inicial de posibilidades?

Desde una posición democrática, es necesario distinguir dos niveles educativos: el primario (o general básico) y el post-primario. Para Guttman (2001), sólo el desarrollo de una teoría democrática de la educación puede fundamentar racionalmente los objetivos de la educación primaria. Tal teoría debe ocuparse de la "reproducción social consciente", es decir, "la forma en que los ciudadanos adquieren (o deberían adquirir) la potestad para influir en la educación que formará los valores políticos, actitudes y formas de comportamiento de los futuros ciudadanos."(30). $\mathrm{Si}$ el ideal democrático de la educación es la "reproducción social consciente", entonces, la educación primaria debe tener como objetivo el desarrollo de la competencia deliberativa, moral y racionalmente fundada, además de "ayudar a los niños a aprender a vivir una vida buena /.../, al enseñarles el conocimiento y la apreciación de la literatura, la ciencia, la historia y los deportes" (73). En otras palabras, "todos los niños deben aprender lo suficiente como para no sólo vivir una vida mínimamente decente, sino también participar de forma efectiva en los procesos democráticos mediante los cuales las elecciones individuales son estructuradas socialmente" y por lo tanto, se debe "evitar aquellas desigualdades que privan a los niños de los logros educativos necesarios para participar en los procesos políticos" $(169)^{[4]}$.

Dos principios confluyen para definir el "estándar de distribución democrática": (i) por el principio del umbral democrático, "ningún niño puede ser privado de la habilidad para participar efectivamente en el proceso democrático", mientras que (ii) el principio de la autorización democrática otorga a la institución democrática la posibilidad de decidir "cuánta educación se debe ofrecer por encima del umbral " (Guttman,2001:172). La extensión del umbral dependerá de las condiciones particulares del país y es materia de decisión democrática, pero las instituciones democráticas no disponen del criterio moral "para decidir si se debe dar o no un nivel adecuado de educación para todos los ciudadanos" (173), es decir, "una vez establecido el umbral no quedaría espacio político para la autoridad democrática discrecional" (174). 
La educación básica, es decir, la transmisión de las habilidades necesarias para la vida en sociedad democrática y de las competencias básicas para poder aprender en el futuro se sitúa y agota en el concepto de "igualdad equitativa de posibilidades" como igualdad en los aprendizajes: todos los individuos deberían tener asegurado el acceso a las competencias básicas (aprendizaje), punto de igualación a partir del cual podrian comenzar a diferenciarse educativamente de acuerdo a sus méritos. Entonces, cualquier desigualdad en el aprendizaje de las competencias básicas, independientemente de su causa, podría considerarse como violación del principio de igualdad equitativa de posibilidades.

La aceptación de esta deducción demanda la satisfacción de los dos condicionales del mencionado principio: las distribuciones desiguales de las capacidades cognitivas y del esfuerzo (aspiración). El primer aspecto no presenta mayor obstáculo: excluyendo algunas situación extremas, todos los individuos en una sociedad son igualmente "educables" en las competencias básicas. Por ello, la desigualdad en este nivel no sería justificable ni siquiera por la desigual distribución de capacidades innatas (dotes naturales).

Para el segundo aspecto - la voluntad del esfuerzo - el propio Rawls ofrece una vía de reflexión a través de la distinción entre el concepto de justicia y las concepciones de la justicia. El concepto de justicia es universal y se refiere a la inexistencia de una "distinción arbitraria" en la atribución de derechos y deberes, al logro de un equilibrio adecuado entre reivindicaciones opuestas correspondientes a ventajas en la vida social. Pero, una sociedad cualquiera se diferencia de otras, en realidad, por la definición que adopte de distinción arbitraria o de distribución adecuada de las ventajas en la sociedad, es decir, por la concepción de justicia (Rawls,1979, sección 1). Veamos cómo podemos aplicar esta distinción a la educación primaria. $\mathrm{Si}$ una sociedad reconoce como distinción arbitraria o conducente a una distribución inadecuada de ventajas, a ciertas cualidades personales pre o extra-escolares que están desigualmente distribuidas según estatus socioeconómicos y que, sin embargo, la escuela exige para lograr los aprendizajes básicos, entonces, una parte importante de la variación del aprendizaje escolar podría interpretarse como violación al principio de la "igualdad de posibilidades", es decir, como injusticia social. El "acceso" no se satisface por la mera permanencia en la escuela durante un determinado período de tiempo, sino que comprende además, el derecho a procesos educativos internos que atiendan a las diferencias pre-escolares, incluidas las culturales. Los procesos de enseñanzaaprendizaje no deben ser discriminatorios. El estudiante tiene el derecho a que la sociedad en general, y la escuela en particular, le ofrezcan la posibilidad real de desarrollar su motivación y de esta forma, su disposición al esfuerzo requerido por la propia escuela (derecho al conocimiento). Las diferencias en la "voluntad de hacer un esfuerzo", cultural y socialmente condicionadas, deben ser atendidas por el sistema educativo como condición para igualar los resultados (aprendizaje de las habilidades básicas).

Educación secundaria (posprimaria) y justicia distributiva. Dado un punto hipotético de igualación en determinados aprendizajes básicos (primaria), incluyendo no sólo conocimientos y habilidades cognitivas, sino también aspiración y esfuerzo, no toda desigualdad educativa de nivel post-primario violará los principios de la justicia distributiva. Ahora la idea de probabilidad es legítima, siempre y cuando sea consecuencia de ventajas proporcionadas por las dotes naturales (capacidad y talento) y la voluntad de emplearlas (aspiración y esfuerzo). El principio de la no-discriminación (o no-exclusión) continúa válido, es decir, la distribución de las probabilidades en los resultados educativos del nivel secundario deben ser independientes de cualquier otro criterio de discriminación (origen social, género, raza, ubicación geográfica). 
A partir de esa idea de probabilidad, el análisis de los determinantes sociales de los resultados educativos adquiere sentido. Para ello, debe acudirse a los denominados "principios distributivos vinculantes", preocupados por el requerimiento de interrumpir o suprimir la "relación entre dos variables" (Van Parijs,1992:213). Referido a las condiciones socioeconómicas familiares del alumno, por ejemplo, el principio distributivo vinculante "negativo" reclama que el origen social del estudiante no afecte la distribución de los resultados educacionales. Tal formulación es generalizable, por un lado, a cualquier otro factor de diferenciación social (género, raza, localización geográfica, etc.) diferente al "mérito" (capacidad, talento, esfuerzo) ${ }^{[5]}$ y por el otro, a tres resultados (probabilidad) educativos:

(i) escolarización, (ii) permanencia (años aprobados) y (iii) aprendizaje efectivo ${ }^{[6]}$.

El foco de interés específico del presente trabajo es justamente el grado en que se viola el "principio vinculante negativo" en el nivel secundario del sistema educativo argentino. En el punto siguiente situamos este tema en relación al concepto de "igualdad de oportunidad educativa" y en la tradición de la investigación empírica a nivel internacional.

\section{B. Oportunidad educativa y antecedentes en la investigación empírica .}

La existencia de una asociación estadísticamente significativa entre origen social del estudiante y su logro escolar está suficientemente probada por innumeras investigaciones realizadas a los largo de las últimas cuatro décadas. El concepto de igualdad de oportunidad educativa (IOE) tiene la virtud de vincular estos resultados con los conceptos centrales de la filosofía política expuesta anteriormente. Coleman fue uno sus principales promotores.

En un estudio seminal, Coleman et.alli. (1966) concluyeron que los insumos escolares tenían poco o ningún efecto sobre las diferencias en el desempeño escolar, es decir, las diferencias en los resultados escolares se debían principalmente a la variación en el origen social del estudiante. Esta constatación indujo a Coleman a proponer que la efectiva igualdad de oportunidad implica "igualdad en aquellos elementos que son efectivos para el aprendizaje" (Coleman,1969:20) o en términos correlacionales, que poseen capacidad predictiva sobre el logro. Pero, demostrada la ineficacia de los insumos escolares (oferta), el planteo inducía a prestar más atención a la igualdad en los resultados (logros) como referente apropiado del concepto de IOE. Por otro lado, los antecedentes familiares explicaron una proporción altamente significativa de las diferencias en los logros; la constatación de que el medioambiente familiar educacionalmente favorable es encontrado más frecuentemente entre estudiantes blancos que entre estudiantes negros, permite concluir que "las diferencias de logros son inequidades de oportunidad por definición." (21). En la situación ideal de la IOE "el medioambiente familiar de los blancos (clase media) y el de los Negros (clase bajas) no producirán efectos sobre el logro..." (23), en cuyo caso, los rendimientos promedios de ambos sectores tenderían a ser iguales.

Posteriormente, Coleman (1975) cuestionó la viabilidad del concepto de IOE en sus versiones extremas, sea como insumo o como resultado (27). La igualdad en el resultado es imposible dada la fuerza del efecto de las diferencias familiares. La igualdad de insumos (i) desconoce las desigualdades justas (discriminación positiva) y por otro lado, (ii) es compatible con muy poca o ninguna escolarización. Debido a ello, reconceptualiza la IOE refiriéndola a la "escuela pública efectiva que conduce en la dirección de oportunidades adultas iguales (28) y por lo tanto, debe "reducir las deficiencias (desventajas) que los niños enfrentan en función de su primer medioambiente sin comprometer al sistema educativo a un fin inalcanzable". (28). Propone entonces, cambiar la condición de 'igualdad' por la de 'reducción de la desigualdad', según la cual "la tarea de la escuela es - al lado de incrementar las 
oportunidades para todos, a través de lo que imparte - reducir el impacto desigualizante en la vida adulta de aquellos diferentes medios ambientes." (29). De esta forma, el concepto de IOE converge con el "principio distributivo vinculante" derivado de la teoría de la justicia distributiva, expuesto anteriormente (ver punto I).

Una investigación de gran envergadura realizada a los pocos años (Jencks, 1972), confirmaba los hallazgos de Coleman, concluyendo que, en la explicación del rendimiento escolar, lo más importante son las características de los propios estudiantes, "todo lo demás - recursos financieros de la escuela, sus políticas, las características de los maestros - es o secundario o completamente irrelevante" (256). Este tipo de conclusión continuó dividiendo a los investigadores. Hacia finales de los años ochenta, revisiones realizadas con el uso de la técnica de meta-análisis se propusieron probar la validez de algunos modelos de determinación del rendimiento (Walberg,1986; Fraser et.alli.,1987), concluyendo que no hay un factor que se sobreponga o que haga desaparecer totalmente los efectos de los otros y por tanto, el rendimiento es explicable a través de una compleja malla de (micro) factores que interactúan entre sí y participan con una pequeña pero importante parcela en la explicación de la variancia total . Los estudios parecen coincidir en que el nivel económico familiar es menos relevante que otras variables familiares, como por ejemplo, su capital cultural o sus actitudes y comportamiento relacionados a la escuela (Ej. el grado de involucramiento en los estudios de los hijos). Pero de todas formas, el balance final de las investigaciones en los países desarrollados es que los antecedentes familiares del estudiante se asocian significativamente con el rendimiento y si bien su peso relativo puede igualar a la de otros factores, difícilmente será inferior.

Tercer Mundo. A mediados de la década del 70, una revisión de algunas investigaciones (Alexander y Simmons, 1975) realizadas en países del Tercer Mundo llegaba a las misma conclusión de Coleman y Jencks: los factores relacionados al origen social del estudiante eran los más relevantes. Sin embargo, basados en la revisión y crítica de los estudios realizados con anterioridad $\mathrm{y}$ en sus propias investigaciones, Heyneman (1976; 1980) y sus colaboradores (Heyneman y Loxley,1982) concluyeron que los errores de medición, los procedimientos metodológicamente cuestionables y la incorrecta especificación de los modelos de rendimiento utilizados explicaban la ambigüedad en los resultados obtenidos por las investigaciones precedentes. La hipótesis básica de Heyneman fue que "cuanto más industrializada es la sociedad, mayor será la probabilidad de que el rendimiento escolar sea afectado por el ambiente socio-económico del estudiante y otras variables no escolares" (Heyneman,1976:205). Las sociedades menos industrializadas, en cambio, son más homogéneas, los estudiantes están menos diferenciados por la aptitud lingüística y la expectativa de escolarización es mucho más baja, y por lo tanto, los antecedentes sociales de los estudiantes ejercen menos influencia sobre el rendimiento. En esta situación, los insumos escolares son "efectivos para el aprendizaje" y por tanto, su igualación conduciría a la IOE, contrariamente a lo establecido por Coleman para la sociedad americana.

En América Latina, una revisión de alrededor de 100 investigaciones realizadas hasta inicios de la década de los años noventa (Wolff, Schiefelbein y Valenzuela,1993), identificó los factores que habían sido estudiados y cuáles de ellos mostraron relaciones consistente con el rendimiento escolar. La conclusión extraída por los autores está inspirada en los trabajos de Heyneman ya mencionados. El estudio concluye que el "insumo educativo contribuye a la adquisición de habilidades cognitivas, independientemente de las características del medio familiar" (28). Sin embargo, esta conclusión no está acompañada con la indicación de cuántos de los estudios revisados controlaron adecuadamente el factor socioeconómico familiar y cuál fue su participación relativa en la explicación de la variación de rendimiento ${ }^{[7]}$. 
Sin desconocer las críticas metodológicas y conceptuales más detalladas a Coleman ${ }^{[8]}$ y a Heyneman y colaboradores, interesa destacar dos objeciones cruciales.

(i) En primer lugar, respecto de las mediciones utilizadas. En los países más desarrollados, altamente estratificados, es más fácil producir indicadores de 'estatus familiar' con alta discriminación, aumentando así la probabilidad de contribución explicativa respecto al logro; los indicadores del sistema educativo, en cambio, son más homogéneos, menos variables ${ }^{[9]}$. En los países del Tercer Mundo, la situación más probable es la inversa. Para Lockheed, Fuller y Nyirondo (1989) las conclusiones divergentes se producen cuando se incluye sólo el aspecto material en la medición de clase social, dejando afuera aspectos culturales, o al usar mediciones que son apropiadas o confiables en los países del primer mundo (educación, ocupación) pero no para los países en desarrollo. Si estos aspectos se tienen en cuenta, se afirma, el efecto del origen social sobre el rendimiento sería mayor ${ }^{[10]}$.

(ii) En segundo lugar, respecto al método de análisis utilizado. Comúnmente, se ha empleado el "mínimo cuadrado ordinario", con el cual no es posible diferenciar ni dimensionar apropiadamente los efectos de las características grupales e individuales, así como tampoco analizar sus interrelaciones. Esta exigencia es clave en los estudios sobre rendimientos escolar, ya que éste es influenciado simultáneamente por factores grupales (escuela, aula) e individuales (aptitud, nivel socioeconómico, sexo, etc). Cuando esta distinción es explícitamente incluida a través de los modelos lineales jerárquico o de niveles múltiples, los resultados pueden sufrir cambios considerables (Riddell,1989; 1997).

En resumen, la estimación del grado de inequidad educativa y de su distribución entre factores escolares y extraescolares puede reflejar más el efecto de ciertos condicionamientos metodológicos que la propia realidad bajo estudio. Si la estimación de la IOE o del grado de violación del "principio distributivo vinculante" incluye tanto factores individuales (nivel socioeconómico, género) como contextuales (composición social de la escuela), el uso de una técnica de análisis que respete, sin sesgos, la estructura jerárquicamente agrupada de los datos analizados se torna crucial.

El estudio del caso argentino resulta particularmente interesante. Al mismo tiempo que la estratificación social compleja ("heterogeneidad social") fue alcanzada hace ya varias décadas, existe una notable variación en la distribución de los recursos y prácticas escolares ("heterogeneidad escolar"). Estudios realizados para el nivel de educación primaria (Cervini,1999;2002) han demostrado que las diferencias entre los rendimientos promedios de las escuelas son notablemente altas - entre $44 \%$ y $52 \%$-, indicando que el uso de la técnica de análisis estadístico "por niveles múltiples" no es sólo recomendable, sino imprescindible.

\section{Concepto operacional de equidad educativa}

Interesa aquí adoptar una definición operacional del concepto de equidad educativa con relación al aprendizaje cognitivo, cuyo indicador es el rendimiento o logro de aprendizaje (puntaje bruto) obtenido por los estudiantes en pruebas estandarizadas ${ }^{[11]}$. El rendimiento varía de estudiante en estudiante. De la misma forma, el rendimiento promedio de los colegios oscila, al igual que lo hace el rendimiento promedio de las Provincias. Entonces, la variación total del rendimiento se expresa como una función de tres componentes: (i) la variación del rendimiento de cada estudiante en torno al rendimiento promedio de su colegio; (ii) la variación del rendimiento promedio de cada colegio en torno al rendimiento promedio de todos los colegios de su misma provincia y (iii) la variación del rendimiento promedio de cada provincia en torno al rendimiento promedio del país.

Según ya fuera expuesto, conocer el grado de violación de la IOE ("principio distributivo vinculante negativo"), requiere estimar las correlaciones entre el rendimiento en la prueba 
y cualquier variable, social o educativa, que estratifique los resultados educativos, propia del alumno individual o del agrupamiento escolar, y diferente de la aptitud (talento), del esfuerzo y de la aspiración del alumno. Pero además, debe incluirse dentro de aquel principio distributivo a la parte de los efectos de la institución educativa y de la Provincia sobre el rendimiento que nada le deben a un posible agrupamiento de talento o de esfuerzo. Para nuestro caso particular, entonces, la (in)equidad educativa se define operacionalmente como la magnitud o intensidad de la asociación entre rendimiento y origen social $y$ género del estudiante (Cuttance,1987), más el grado de variación inter-colegio e inter-Provincia del rendimiento no debida al agrupamiento de talento o esfuerzo. El origen social del estudiantes puede desdoblarse en dos dimensiones: el capital económico y el capital cultural; en este último, además, es posible distinguir entre la forma "incorporada" y la "objetivada" ${ }^{[12]}$.

A través de la técnica de análisis utilizada en este trabajo (modelo de niveles múltiples) es posible, en primer lugar, evaluar el 'efecto colegio' y el 'efecto Provincia' sobre la distribución del rendimiento, es decir, si las variaciones de los rendimientos promedios institucionales y provinciales son estadisticamente significativas; en segundo lugar, determinar la fuerza de la relación entre el rendimiento y las variables socioeconómicas y el género, tanto en general como en cada uno de los niveles de agregación (inequidad institucional $e$ inequidad Provincial); en tercer lugar, distinguir el efecto de tales factores en su forma individual (familia del estudiante) del ejercido como "contexto".

Por otro lado, la forma positiva del "principio distributivo vinculante" clama por la distribución de los aprendizajes de acuerdo al "mérito" (capacidad y esfuerzo). En este caso, el análisis empírico consiste en determinar qué proporción de la desigualdad en el rendimiento de los estudiantes se debe a los factores "justos" (Ej. antecedentes académicos del estudiante). En el presente estudio no se dispone de ninguna medición específica de tales factores, pero se incluye un indicador "proxy": el antecedente de repitencia de año, que expresa en parte, la capacidad o aptitud académica del alumno ${ }^{[13]}$.

\section{Objetivos y Metodologia}

Objetivos. Los propósitos del trabajo atienden al concepto de equidad educativa expuesto anteriormente, en el marco de la teoría de la justicia distributiva. Al finalizar el análisis se espera haber respondido las siguientes preguntas, respecto de los logros en Matemática y Lengua del secundario en Argentina:

1. ¿Cuál es el probable grado (máximo) de (in)equidad producida por el agrupamiento escolar (inequidad institucional) y provincial (inequidad geográfica)? Es decir, determinación del 'efecto escuela' y del 'efecto Provincia';

2. ¿Cuál es el grado de (in)equidad producido por factores socioeconómicos y el género? ¿Cuál es el efecto de la repitencia escolar? ¿Cuáles variables tienen más peso, las individuales (familia) o las contextuales?

3. ¿El grado de (in)equidad educativa varía entre los colegios y las Provincias?

Los datos. Se analizan datos que provienen del Cuestionario del Alumno y de las pruebas de Matemática y de Lengua aplicadas a los estudiantes del último año del secundario durante el Censo Nacional de Finalización del Nivel Secundario de 1998, realizado por el Ministerio de Cultura y Educación de la Nación de Argentina. La evaluación se realizó a finales del año lectivo. Se incluyen solamente los estudiantes que dieron las pruebas de Matemática y/o Lengua y que además, hubiesen respondido el cuestionario correspondiente. Finalmente, fueron excluidos los colegios con información completa para menos de 10 estudiantes. Con estas condiciones, el archivo de Matemática queda constituido por 161.054 estudiantes en 3.322 
colegios y el de Lengua por 155.419 estudiantes en 3.282 colegios.

Variables. Las variables individuales analizadas son:

a) Variables criterio: puntaje (bruto) obtenido en sendas pruebas estandarizadas de Matemática (z_matema) y Lengua (z_Lengua);

b) Capital económico:

- (z_bienes): disponibilidad de 17 bienes de uso durable y servicios en el hogar;

c) Capital cultural:

- (z_educa): nivel educativo del padre y de la madre;

- (z_cultur): disponibilidad de libros en el hogar;

- (z_dida): disponibilidad de libros, fichas y apuntes escolares;

d) (femenino): género del estudiante (variable "dummy":femenino; base: masculino);

e) (repite): repitencia del estudiante (variable "dummy" :repitente; base: no repitente).

Todas las variables han sido estandarizadas, con excepción de (femenino) y (repite).

Las variables contextuales (colegio y Provincia) del nivel socioeconómico se forman con el promedio de cada variable individual del estudiante, y las de género y repitencia se definen como proporciones. El final de la sigla indica el nivel de agrupamiento. La agregación a nivel colegio se indica como "col". Así por ejemplo, (bien_col) es el promedio de (z_bienes) en el colegio y (feme_col) es la proporción de estudiantes del sexo femenino en el colegio.

Técnica de análisis. Para el análisis de las relaciones entre el rendimiento y las diferentes variables consideradas, se utilizó la técnica de "análisis estadístico por niveles múltiples" (Aitkin y Longford, 1986; Bryk y Raudenbush,1992; Goldstein,1987). Esta es una técnica correlacional adecuada para analizar variaciones en las características de los individuos $(\mathrm{Ej}$. rendimiento en matemática) que son miembros de un grupo (Ej. colegio) que a su vez, hace parte de otra agregación (Ej. Provincia), o sea, mediciones que forman parte de una estructura agrupada $y$ jerárquica. La técnica permite la descomposición de la variación de una variable (Ej. rendimiento) en sus componentes "dentro del grupo" (intra-colegio; intra-Provincia) y "entre grupo" ('inter-colegio'; 'inter-Provincia') y el análisis de la asociación entre variables en esos diferentes niveles de agregación. El modelo se compone de una Parte Fija, con los parámetros que definen una línea promedio para todos los estudiantes de todos los colegios de todas las Provincias, y de una Parte Aleatoria, que muestra en cada nivel de agregación, la estimación de la variación de los parámetros determinados en la parte fija. La principal ventaja de esta técnica es que modela simultáneamente los diferentes niveles de variación (por ejemplo, estudiante, colegio y Provincia), permitiendo, por tanto, saber qué proporción de la variación del rendimiento escolar se debe principalmente a características del estudiante, del colegio y de la Provincia. Para estimar la probabilidad del efecto de las variables se usa el test de la razón de máxima verosimilitud.

Niveles de agregación. Los datos permiten definir modelos con tres niveles de agrupamiento: el estudiante (nivel 1), el colegio (nivel 2) y la Provincia ${ }^{[14]}$ (nivel 3).

Estrategia metodológica. El ordenamiento secuencial del análisis responde a los objetivos propuestos y a la técnica de análisis: se comienza con la partición de la varianza del rendimiento por niveles $\left(1^{\circ}\right.$ paso), para continuar modelando las correlaciones entre el rendimiento y los indicadores individuales del estudiante ( $2^{\circ}$ paso); a continuación se agregan los indicadores del contexto institucional $\left(3^{\circ}\right.$ paso), y los referidos al género y la repitencia ( $4^{\circ}$ paso) para finalmente, sumar también los indicadores del contexto provincial ( $5^{\circ}$ paso). Finalmente $\left(6^{\circ}\right.$ paso $)$, se analizan las 
variaciones de la (in)equidad educativa a nivel institucional y provincial.

\section{Resultados y análisis}

\section{A. Las desigualdades en la escolarización y la permanencia.}

En Argentina ya se ha logrado la escolarización universal en la educación primaria, al menos si se consideran sólo los primeros 7 años de educación. Este no es, sin embargo, el caso para la educación secundaria. En un análisis de datos estadísticos recientes (SIEMPRO, 2000), provenientes de un relevamiento muestral urbano ${ }^{[15]}$, se informa que la cobertura total secundaria de la población de 13 a 17 años es de $82 \%{ }^{[16]}$, pero distribuida muy desigualmente: mientras que para el $1^{\circ}$ quintil de ingreso ("el $20 \%$ más pobre") apenas sobrepasa el $70 \%$, en el último quintil ("los más ricos") es superior a $97 \%$. Además, de la población más pobre de 15 a 24 años que había comenzado el colegio secundario, casi el $30 \%$ lo abandonó sin terminarlo, situación que alcanza a sólo el 6,6\% de la población más favorecida económicamente. Como consecuencia, el $24,4 \%$ de los jóvenes de 18 a 24 años en el $1^{\circ}$ quintil han completado la secundaria, porcentaje que llega al $76 \%$ entre esa población en el quintil de más alto ingresos.

\section{B. Las desigualdades en el rendimiento}

Las desigualdades de las tasas de escolarización y permanencia del secundario tornan evidente que el análisis de la distribución de los aprendizajes entre los estudiantes del último año de ese nivel supone un alto grado de selectividad socioeconómica, más especificamente, económica. Seguramente, cierta proporción de los que no están deberían estar si se atendiese exclusivamente a su capacidad, talento, aspiración o aún, predisposición para el esfuerzo. En consecuencia, la inequidad en la distribución de los rendimientos debe considerarse sólo una parte de la inequidad educativa total en el nivel secundario del sistema educativo argentino. $1^{\circ}$ paso: Partición inicial de la varianza en los tres niveles de agregación (modelo "vacio"). Se estiman la variación alrededor de la media global del rendimiento (Parte fija) y simultáneamente, las variaciones en cada nivel de agregación (Provincia, colegio y estudiante) (Parte aleatoria). ), sin ningún predictor ("modelo vacío"). Los resultados de este paso permiten responder provisoriamente al primer objetivo.

Para calcular el rendimiento promedio global y las tres variaciones ('inter-Provincia', 'intercolegio' e "inter-estudiante"), se regresa el rendimiento de cada estudiante $\left(\right.$ rend $\left._{\mathrm{ijk}}\right)$ sobre una constante (cons) que asume un valor $=1$ para todos los estudiantes. Si se toman como ejemplo los resultados en Matemática, formalmente la operación se expresa así:

$$
\begin{aligned}
& z_{-} \text {matema } \mathrm{i}_{i k} \sim \mathrm{N}(X B, \Omega) \\
& z_{-} \text {matema } a_{i k}=b_{0 i j k} \text { cons } \\
& b_{0 j k}=-0,339(0,084)+v_{0 k}+u_{0 j k}+e_{0 j k} \\
& {\left[v_{0 k}\right] \sim \mathrm{N}\left(0, \Omega_{v}\right): \Omega_{v}=[0,134(0,044)]} \\
& {\left[u_{0 ; k}\right] \sim \mathrm{N}\left(0, \Omega_{u}\right): \Omega_{u}=[0,346(0,009)]} \\
& {\left[e_{0 j k}\right] \sim \mathrm{N}\left(0, \Omega_{e}\right): \Omega_{e}=[0,559(0,002)]} \\
& -2 * \log (i k e)=374049,300
\end{aligned}
$$

donde $\quad z$ matema $a_{\mathrm{ijk}}$ es el rendimiento (estandarizado) en Matemática del estudiante $i$ en el colegio $j$ de la Provincia $k$; (cons) es una constante igual a $1 ; \beta_{0}$ es el rendimiento promedio estimado (Parte fija); $\mathrm{e}_{0 \mathrm{ijk}}$ es el "residuo" a nivel estudiante (nivel 1), para el $i$ ésimo estudiante en el j-ésimo colegio de la $k$ ésima Provincia; $\mu_{0 \mathrm{jk}}$ es el residuo a nivel colegio (nivel 2), para el j-ésimo colegio de la $k$ ésima Provincia, y $v_{0 \mathrm{k}}$ es el residuo de nivel 3 (Provincia). Las últimas tres variables $\left(\mathrm{e}_{0 \mathrm{ijk}}, \mu_{0 \mathrm{jk}}\right.$ $\left.\mathrm{y} \quad v_{0 \mathrm{k}}\right)$ son cantidades aleatorias, con una media $=0$, no correlacionadas y con distribución normal (Parte aleatoria). Por lo tanto, podemos estimar sus varianzas, indicadas como $\sigma_{\mathrm{e}}, \sigma_{\mu} \mathrm{y}$ $\sigma_{v}$, respectivamente, quedando así especificadas las proporciones de la variación total del rendimiento que se deben a las diferencias entre 
las Provincias, entre los colegios y entre los estudiantes. Obsérvese que la suma de las proporciones sobrepasa levemente a la unidad $(1,039)$; esto se debe a las fluctuaciones de muestreo. Las dos primeras representan principalmente, aunque no exclusivamente, el peso que tienen las características grupales en la explicación de las variaciones totales del rendimiento escolar en Matemática. El valor del test de máxima verosimilitud es 374049,3.

De los resultados (Cuadro 1) se infiere que:

a) La varianza 'inter-Provincia' de los puntajes promedios provinciales resultó estadísticamente significativa, es decir, las Provincias se diferencian significativamente entre sí respecto del nivel de rendimiento que alcanzan sus alumnos hacia el final del nivel secundario. La variación detectada debe entenderse como una consecuencia de la selectividad geográfica o regional del sistema educativo. Nótese que tal segmentación es más pronunciada en Matemática $(13,4 \%)$ que en Lengua $(10,2 \%)$; es decir, los resultados obtenidos por los estudiantes en Lengua son regionalmente más homogéneos que los de Matemática;

\section{Distribución porcentual de la variación del rendimiento por materia, según nivel de agregación}

\begin{tabular}{|c|c|c|}
\hline Nivel de agregación & Matemáticas & Lengua \\
\hline Provincia & 13,4 & 10,2 \\
\hline (err.std.) & $(0,044)$ & $(0,034)$ \\
\hline Colegio & 34,6 & 32,8 \\
\hline (err.std.) & $(0,009)$ & $(0,009)$ \\
\hline Estudiante & 55,9 & 62,4 \\
\hline (err.std.) & $(0,002)$ & $(0,002)$ \\
\hline Total & $\mathbb{1 0 3 , 9}$ & $\mathbf{1 0 5 , 4}$ \\
\hline
\end{tabular}

b) La varianza 'inter-colegio' de los puntajes promedios de los colegios es altamente significativa. Alrededor del $34,6 \%$ de la variación del rendimiento en Matemática y del $32,8 \%$ en Lengua se debe a diferencias entre los establecimientos educativos. Es decir, los colegios se diferencian significativamente entre sí respecto al nivel de aprendizaje que alcanzan sus egresados. Alrededor de un tercio de las desigualdades en los niveles de aprendizaje finales de Matemática y Lengua son atribuibles a la existencia de una fuerte segmentación institucional en la educación secundaria;

c) Finalmente, la variación "entre-estudiante"(o intra-colegio) constituye casi el $56 \%$ de la variación total del rendimiento en Matemática y más del 62\% de la de Lengua. La mayor variación en Lengua significa que, en general, los estudiantes de un mismo colegio tienden a obtener aprendizajes más homogéneos en Matemática.

En resumen, los resultados obtenidos permiten inferir que vivir en una Provincia o asistir a un colegio determinado implica una mayor (menor) probabilidad de acceder a un nivel de aprendizaje significativamente más alto (bajo) que el esperado en otras provincias y/o colegios. Se observó además, que el mayor peso en las desigualdades se debe a las instituciones educativas. Aparentemente, entonces, la segmentación institucional es 
Cervini, Rubén (2002). Desigualdades Socioculturales en el Aprendizaje de Matemática y Lengua de la Educación Secundaria en Argentina. RELIEVE:, v.8, n. 2, p. 126-148.

http://www.uv.es/RELIEVE/v8n1/RELIEVEv8n2 1.htm

mucho más acentuada que las diferencias provinciales.

$2^{\circ}$ paso: Rendimiento $y$ origen social del estudiante: indicadores individuales. Ahora se determina el grado de inequidad educativa debido al origen social del estudiante individual, es decir, se evalúa el grado de asociación del rendimiento con cada medición individual de nivel socioeconómico, actuando todas ellas simultáneamente. Para ello, se debe incluir a todos los indicadores en el modelo "vacío" y analizar las estimaciones que resulten para cada uno de ellos. Los resultados (Cuadro 2) permiten concluir que:

a) El indicador de capital económico (z_bienes) no es estadísticamente significativo en Lengua y, aunque en Matemática sí lo es, su magnitud es claramente reducida;

b) En cambio, los tres indicadores de capital cultural son nítidamente significativos, es decir, cada uno de ellos tiene un peso propio, sugiriendo que indican aspectos diferenciables en la explicación de las diferencias en el nivel de aprendizaje. Nótese que (z_libros) es el de mayor peso en ambas materias;

c) El efecto del conjunto de indicadores se detecta principalmente por el descenso de la variación 'inter-colegio' no-explicada: de $34,6 \%$ y $32,8 \%$ en los modelos "vacíos" de Matemática y Lengua, a 29,5\% y 27,2\%, respectivamente;

\begin{tabular}{|c|c|c|c|c|}
\hline \multicolumn{5}{|c|}{$\begin{array}{l}\text { 2. Efecto de los indicadores socio-económicos individuales del alumno y } \\
\text { variación (\%) no explicada en cada nivel de agregación. }\end{array}$} \\
\hline \multirow[b]{2}{*}{ Indicadores } & \multicolumn{2}{|c|}{ Matemáticas } & \multicolumn{2}{|c|}{ Lengua } \\
\hline & Estimación & $\begin{array}{c}\text { Error } \\
\text { estándar }\end{array}$ & Estimación & $\begin{array}{c}\text { Error } \\
\text { estándar }\end{array}$ \\
\hline $\begin{array}{c}\text { Modelo } \\
\text { Vacio }\end{array}$ & \multicolumn{2}{|c|}{374049,3} & \multicolumn{2}{|l|}{377610,7} \\
\hline z_bienes & 0,009 & 0,002 & ns & ---- \\
\hline z_educa & 0,076 & 0,003 & 0,064 & 0,003 \\
\hline z_libros & 0,081 & 0,002 & 0,104 & 0,002 \\
\hline z_dida & 0,022 & 0,002 & 0,063 & 0,002 \\
\hline \multicolumn{5}{|l|}{ Nivel } \\
\hline Provincia & 10,8 & 0,036 & 7,3 & 0,025 \\
\hline Colegio & 29,5 & 0,008 & 27,2 & 0,007 \\
\hline Estudiante & 54,8 & 0,002 & 60,7 & 0,002 \\
\hline Test & \multicolumn{2}{|c|}{$370282,7^{*}$} & \multicolumn{2}{|c|}{$372854,8^{*}$} \\
\hline \multicolumn{5}{|c|}{$\left(^{*}\right)$ Prob. $\leq 0.001$} \\
\hline
\end{tabular}

d) La variación estimada para el nivel de Provincia también ha experimentado un descenso importante en ambas Materias.

De (a) y (b) se infiere que la inequidad en la distribución de saberes escolares secundarios está más relacionada con la distribución del capital cultural que con el económico. De (c) y (d) se infiere que indicadores definidos a nivel individual del estudiante producen efectos casi exclusivamente en un nivel de agregación superior, es decir, en las desigualdades de 
Cervini, Rubén (2002). Desigualdades Socioculturales en el Aprendizaje de Matemática y Lengua de la Educación Secundaria en Argentina. RELIEVE:, v.8, n. 2, p. 126-148.

http://www.uv.es/RELIEVE/v8n1/RELIEVEv8n2_1.htm

rendimiento 'inter-colegio' e 'inter-Provincia'. Esto sugiere que la composición de los grupos de estudiantes (colegios) respecto de los indicadores socioeconómicos no es exactamente idéntica en todos ellos y que una parte substancial de la variación del rendimiento se relaciona con tal diferencia de composición. Se puede adelantar la conclusión de que este comportamiento de los datos refleja, de hecho, la fuerte segmentación social del sistema educativo argentino.

$3^{\circ}$ paso: Rendimiento, origen social del estudiante y contexto socioeconómico institucional. En este paso se evalúa el grado de asociación de cada medición individual y contextual (institución) con el rendimiento, actuando simultáneamente. Para ello, a los modelos expuestos en el Cuadro 2 se les incorporan los indicadores del contexto institucional. Los resultados se presentan en el Cuadro 3: a) el indicador ( $z$ bienes) ahora se muestra no significativo también en Matemática;

b) la medición del contexto económico (bien col) tampoco es significativo en ambas materias;

c) todos los indicadores individuales y contextuales del capital cultural son significativos y es posible identificar el efecto propio de cada uno de ellos;

d) el indicador con mayor capacidad predictiva en ambas materias (libro_col) se refiere al contexto, comportamiento que tiende a confirmar la hipótesis de una pronunciada segmentación cultural en la distribución de los aprendizajes secundarios,

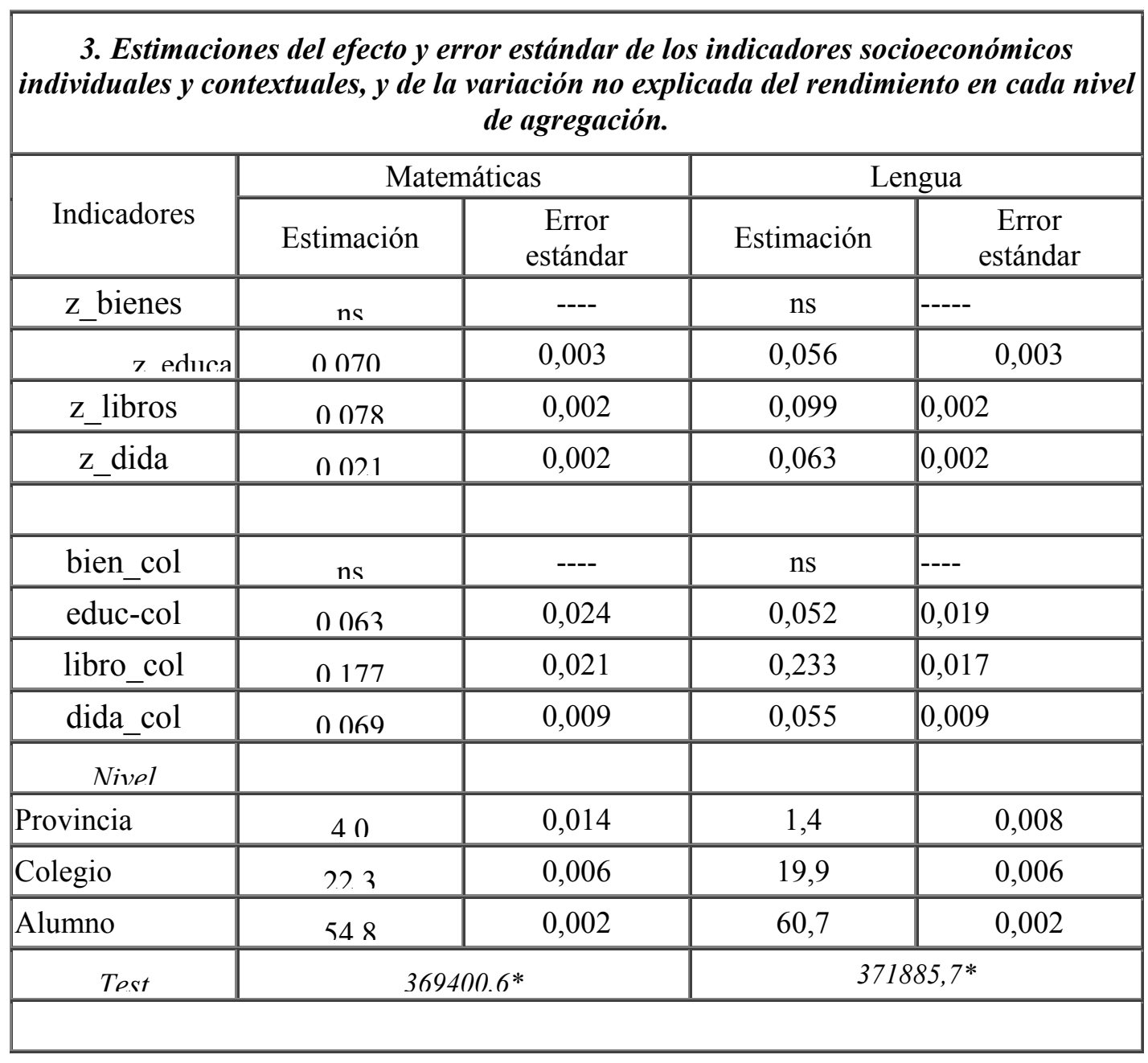


e) el nivel educativo de los padres, tanto individual como contextual, es mejor predictor de los rendimientos en Matemática que en Lengua, mientras que con los indicadores de 'acceso a bienes culturales' prácticamente sucede lo contrario;

f) el conjunto de indicadores ha conseguido que la variación 'inter-colegio' descienda de 34,6\% y $32,8 \%$ en los modelos "vacíos" de Matemática y Lengua, a 22,3\% y 19,9\%, respectivamente, lo cual significa que estos indicadores 'explican' alrededor del 35\% de las diferencias de rendimiento promedio en Matemática entre los colegios y casi el $40 \%$ de las referidas a Lengua;

g) asimismo, las variaciones 'inter-Provincia' no-explicadas han descendido a $4,8 \%$ en Matemática y son prácticamente nosignificativas en Lengua.

En resumen, la principal fuente de inequidad en el aprendizaje de los saberes escolares del secundario es el capital cultural y tal inequidad se procesa principalmente a través de la segmentación cultural institucional y regional. Es posible distinguir claramente los efectos de las dos formas del capital cultural (capital 'incorporado' y 'objetivado') ${ }^{[17]}$. Pero por otra parte, en ambas materias la variación del rendimiento promedio de los colegios y las
Provincias continúan siendo significativa. Si bien las desigualdades de capital cultural (individual y contextual) explican una gran parte de las diferencias en los rendimientos promedios institucionales, mayores son las desigualdades que han quedado sin explicar $22,3 \%$ en Matemática y 19,9\% en Lengua. Una parte de ellas se debe seguramente a la heterogénea distribución institucional y provincial de factores escolares eficaces, fuentes también de injusticia distributiva educativa.

$4^{\circ}$ paso: Rendimiento, género $y$ antecedentes académicos. Corresponde ahora, evaluar las relaciones del género y del antecedente de repitencia del estudiante con el rendimiento en ambas materias. Para ello, procedemos a incluir en los modelos anteriores (Cuadro 3) las dos mediciones correspondientes, en sus formas individual - (femenino) y (repite) -, tratadas con la técnica de variable "muda" ("dummy"), y contextual (proporción de mujeres y repitientes). Los resultados de esta operación (Cuadro 4) indican que:

(salto a la página siguiente) 
Cervini, Rubén (2002). Desigualdades Socioculturales en el Aprendizaje de Matemática y Lengua de la Educación Secundaria en Argentina. RELIEVE:, v.8, n. 2, p. 126-148.

http://www.uv.es/RELIEVE/v8n1/RELIEVEv8n2_1.htm

\begin{tabular}{|c|c|c|c|c|}
\hline \multicolumn{5}{|c|}{$\begin{array}{c}\text { 4. Estimaciones del efecto y error estándar de los indicadores socioeconómicos } \\
\text { individuales y contextuales, del género y la repitencia, y de la variación no explicada } \\
\text { del rendimiento en cada nivel de agregación. }\end{array}$} \\
\hline \multirow{2}{*}{ Indicadores } & \multicolumn{2}{|c|}{ Matemática } & \multicolumn{2}{|c|}{ Lengua } \\
\hline & Estimación & Error estándar & Estimación & Error estándar \\
\hline z_bienes & ns & ---- & ns & ---- \\
\hline z_educa & 0,060 & $(0,002)$ & 0,059 & $(0,003)$ \\
\hline z_libros & 0,076 & $(0,002)$ & 0,096 & $(0,002)$ \\
\hline z_dida & 0,022 & $(0,002)$ & 0,035 & $(0,002)$ \\
\hline femenino & $-0,058$ & $(0,004)$ & 0,233 & $(0,005)$ \\
\hline repiten & $-0,231$ & $(0,005)$ & $-0,263$ & $(0,005)$ \\
\hline bien_col & ns & ---- & ns & ---- \\
\hline educ-col & ns & ---- & ns & ---- \\
\hline libro_col & 0,173 & $(0,020)$ & 0,183 & $(0,019)$ \\
\hline dida_col & 0,050 & $(0,009)$ & 0,046 & $(0,008)$ \\
\hline feme_col & $-0,063$ & $(0,008)$ & 0,025 & $(0,007)$ \\
\hline repi_col & -0133 & $(0,009)$ & $-0,111$ & $(0,008)$ \\
\hline \multicolumn{5}{|l|}{ Nivel } \\
\hline Provincia & 0,048 & $(0,017)$ & 0,021 & $(0,008)$ \\
\hline Colegio & 0,193 & $(0,005)$ & 0,168 & $(0,005)$ \\
\hline Alumno & 0,539 & $(0,002)$ & 0,585 & $(0,002)$ \\
\hline Test & \multicolumn{2}{|c|}{$366529,3 *$} & \multicolumn{2}{|c|}{$365731,0^{*}$} \\
\hline \multicolumn{5}{|c|}{$\left(^{*}\right)$ Prob. $\leq 0.001$} \\
\hline
\end{tabular}

a) las mujeres obtienen más bajos rendimientos que los hombres en Matemática; sucede lo contrario en Lengua. Aunque ambas distancias son estadísticamente significativas, la de Lengua es notablemente más pronunciada; b) en cambio, el indicador de 'historia académica' (repitencia) del estudiante individual tiene un efecto fuerte y similar en ambas asignaturas; 
c) la expresión contextual de ambas variables adicionan una contribución propia a la explicación de las desigualdades en el rendimiento en ambas materias y en el mismo sentido que los indicadores individuales: a mayor proporción de repitientes en el colegio, menores rendimientos promedio y a mayor proporción de mujeres más bajos rendimientos promedios en Matemática y más altos en Lengua;

d) al incluir la repitencia, las estimaciones de los indicadores de capital cultural experimentan, en general, un descenso leve y continúan siendo estadísticamente significativas; es decir, el capital cultural ejerce un efecto directo significativo sobre el rendimiento, adicional al que puede estar ejerciendo a través de la repitencia del estudiante;

e) se verificó una excepción a esa tendencia general: con la inclusión de la 'proporción de repitientes' (repi col) se desvanece el efecto del nivel educativo contextual (educ_col). Entonces, los efectos de ambas mediciones contextuales sobre el rendimiento se superponen; la proporción de estudiantes repitientes acompaña al 'capital cultural incorporado' contextual y el efecto de éste se efectiviza a través de los 'antecedentes académicos' contextuales;

f) finalmente, se observa que las nuevas variables han ocasionado descensos principalmente en la varianza no-explicada del nivel colegio; de esta forma, vuelve a manifestarse el carácter segmentado del sistema educativo secundario.

\section{$5^{\circ}$ paso: Rendimiento y contexto provincial. En} este paso se verifica si los indicadores expresados como promedios provinciales dan cuenta de la varianza residual a nivel Provincia. Para ello se los incluyó en el modelo anterior y se evaluaron los cambios acontecidos. Se constató que (i) tanto el indicador económico (bie prov), como el de nivel educativo (edu_prov) no son explicativos de las diferencias interprovinciales; (ii) los indicadores de 'capital cultural objetivado' son predictores estadísticamente significativos cuando actúan individualmente; (iii) pero, actuando conjuntamente, el indicador 'posesión de libros en el hogar' es más influyente en Lengua, mientras que para Matemática, el más importante es el referido a los textos escolares. La adición de estos efectos produce una caída de la variación 'inter-Provincia' a $1 \%$ en Lengua y a 2,1\% en Matemática. Es decir, el conjunto de indicadores analizados en este trabajo "explican" prácticamente la totalidad de las diferencias de rendimiento promedio entre las provincias del pais, tanto en Lengua como en Matemática. Con este último análisis se completa la respuesta a la segunda pregunta de investigación.

$6^{\circ}$ paso: Aleatorización del efecto del capital cultural. Hasta aquí se ha supuesto que la intensidad de la asociación entre rendimiento y los factores estudiados es similar en todos los colegios. Sin embargo, ella puede variar, es decir, pueden existir diferencias en el grado de (in)equidad institucional, reflejando disparidades en la capacidad institucional para compensar el efecto del origen sociocultural del estudiante sobre su aprendizaje. Para evaluar esta posibilidad se debe permitir que aquella correlación varíe, es decir, se debe aleatorizar el efecto a nivel colegio. En el Cuadro 5 se presentan los resultados en el test de máxima verosimilitud:

a) los efectos de todos los indicadores varían significativamente entre colegios; entonces, la multiplicidad de espacios institucionales está caracterizada por diferencias notables en la capacidad redistributiva;

b) la diferencia hombre/mujer es la que más varía, tanto en Matemática como en Lengua (en ambas asignatura posee el menor valor del test);

c) el efecto de los indicadores con mayor poder "explicativo" (posesión de libros en Lengua y de los textos didácticos en matemática) es el más homogéneo a lo largo del conjunto de instituciones. 
Cervini, Rubén (2002). Desigualdades Socioculturales en el Aprendizaje de Matemática y Lengua de la Educación Secundaria en Argentina. RELIEVE:, v.8, n. 2, p. 126-148.

http://www.uv.es/RELIEVE/v8n1/RELIEVEv8n2 1.htm

\begin{tabular}{|r|c|c|}
\hline \multicolumn{2}{|c|}{$\begin{array}{r}\text { 5. Valor del test de máxima verosimilitud para modelos con } \\
\text { aleatorización a nivel colegio }\end{array}$} \\
\hline \multirow{2}{*}{ Indicadores } & \multicolumn{2}{|c|}{ Test de máxima verosimilitud } \\
\cline { 2 - 3 } & Matemática & Lengua \\
\hline Modelo final & 366516,6 & 365718,9 \\
\hline femenino & 366045,4 & 365133,3 \\
\hline z_educa & 366294,9 & 365578,3 \\
\hline z_libros & 366379,7 & 365644,3 \\
\hline z_dida & 366433,1 & 365560,6 \\
\hline
\end{tabular}

\section{Conclusiones}

La fuerza de la asociación entre el origen social del alumno o su género, por un lado, y el acceso y/o permanencia escolar, por el otro, denuncia el grado de injusticia educativa existente en un país. En Argentina, la escolarización y permanencia universal en el nivel secundario no parece alcanzable a corto plazo. Los 'excluidos' son los sectores más pobres de la sociedad. Sobre esta exclusión poco o nada puede pedírsele al sistema educativo. A ella debe responderse con políticas macroeconómicas y sociales adecuadas ${ }^{[18]}$. Pero la injusticia educativa, como negación del principio distributivo vinculante o de la igualdad de oportunidad educativa, también se encuentra entre los 'incluidos'. A través del análisis de los datos del Censo Nacional de Finalización del Nivel Secundario de 1998 se pretendió develar su importancia, determinando los niveles de desigualdad en los aprendizajes de Matemática y de Lengua debidos (i) al agrupamiento escolar y provincial y (ii) a la incidencia de factores socioeconómicos extra-escolares.

i. En base a una revisión de más de 40 investigaciones recientes, realizadas en diferentes países del Tercer Mundo y que han usado la técnica de modelos lineales jerárquicos' (niveles múltiples), Riddell (1997) concluye que con mayor frecuencia las diferencias "entre colegios" son menores que las disparidades "entre estudiantes", es decir, "la influencia del hogar es mayor que la de la escuela" (185).Para el nivel secundario, el promedio de las diferencias "entre estudiantes" estimadas es levemente inferior a 55\% para Matemática y alrededor del $60 \%$ en Lengua. Los datos analizados para el caso Argentino se ajustaron nítidamente a esta tendencia general.

ii. Los datos permitieron determinar las variaciones del rendimiento "entre Provincia y "entre colegio". A través de sus comportamientos se infiere que las probabilidades de aprendizaje en el nivel secundario están más fuertemente vinculadas a la institución educativa a la que se accede que a la Provincia en que se vive.

iii. No es el capital económico de la familia del alumno o del contexto escolar el que más importa a la hora de dimensionar la injusticia en la distribución de los aprendizajes. Ese capital define la inclusión-exclusión inicial, es decir, determina las probabilidades de acceso y permanencia. A partir de allí, es el capital cultural familiar y contextual el que moldeará el perfil de la distribución del logro escolar. Y dentro de este acotamiento, más 
importante que el "volumen" de capital cultural 'incorporado' es su activación (Lareau,1987), indicada indirectamente por la posesión de capital cultural objetivado y relacionado al sistema educativo.

iv. El análisis de los datos demostró que la incidencia de la 'herencia cultural familiar' del alumno individual opera a través del agrupamiento en instituciones escolares. No es observando cada colegio o cada aula que se tornará más evidente la relación entre 'capital cultural' y éxito escolar. Los 'culturalmente iguales' tienden a estar institucionalmente juntos y por tanto, sus diferencias de logro deben ser explicadas por otras características personales. Consistentemente, los indicadores del 'contexto cultural institucional' muestran una capacidad predictiva del rendimiento notablemente mayor que sus homónimos del alumno individual. En otras palabras, la injusticia educativa opera principalmente a través de la segmentación sociocultural del entramado institucional.

v. Al mismo tiempo, y sin alterar la validez de la conclusión anterior, se observó que el grado de equidad institucional varía significativamente: los colegios difieren respecto del grado en que el origen social del estudiante incide en la distribución de los aprendizajes; algunos consiguen 'compensar' las desigualdades de origen de sus estudiantes más eficazmente que otros.

vi. Pero, una proporción importante de la desigualdad educativa total - alrededor del 18\% - se debe a otros tipos de factores. Parte de esa inequidad podría provenir del efecto de factores propiamente escolares, alterables $\mathrm{y}$ pasibles de ser distribuidos equitativamente. Y de esta posibilidad, el sistema sí tiene responsabilidad pública. De allí la importancia de investigarlos. Las diferencias interprovinciales parecen no merecer esta atención: fueron casi totalmente explicadas por las diferencias en su composición sociocultural.

vii. La diferenciación de género en los niveles de aprendizaje confirma las constataciones recurrentemente encontrada en los estudios internacionales: la mujeres logran mejores niveles en Lengua y los hombre en Matemática. Fue probado, sin embargo, que esa distancia es visiblemente más abultada en Lengua, a favor de las mujeres, que la existente en Matemática a favor de los hombres. Al igual que lo registrado para el capital cultural, el grado de equidad institucional respecto al género varía significativamente: los colegios difieren respecto al grado en que el género del estudiante incide en la distribución de los aprendizajes, es decir, en algunos, la distancia hombre/mujer es menor que en otros.

En Argentina, la cobertura de la educación media ha continuado creciendo durante la última década, a pesar de una alteración regresiva en la distribución del ingreso y un aumento consecuente en los niveles de pobreza. Esas condiciones, agravadas por las restricciones en los recursos públicos disponibles (menor gasto per capita), conducen a una expansión de la oferta que reproduce y consolida la injusticia educativa por medio de la segmentación institucional. Es decir, los nuevos ingresantes, población proveniente de estratos anteriormente excluidos, son incorporados en un sistema que distribuye desigualmente las probabilidades de aprendizaje. Es probable que este proceso de segmentación se haya realizado y continúe haciéndose, a través de tres mecanismos interrelacionados de estructuración: la diferenciación público/privado, las pronunciadas diferencias institucionales en infraestructura y recursos didácticos y la fuerte homogeneidad en la composición social y cultural de los colegios, en base a diferentes niveles de segregación residencial. En futuros estudios se intentará conocer cuál es el peso relativo de cada uno de estos factores de segmentación.

Según un documento reciente editado por la CEPAL (Ocampo, 2000), la expansión de la educación secundaria "eleva la competitividad 
sistémica del conjunto de la sociedad" ya que posibilita la incorporación del progreso técnico y "el uso intensivo de la información y el conocimiento" (110), y la forma económicamente más conveniente de realizar esa expansión es a través de la "inversión educacional oportuna, es decir, realizada en el período de edad correspondiente" (110). Entre los grandes desafíos actuales, figuran no sólo "llegar dentro de los plazos más cortos posibles a una cobertura universal de la educación secundaria" sino también "promover una reforma educativa que permita igualar las oportunidades de acceso a una educación de calidad..." (95). La crudeza de los datos analizados indican que para poder caminar en ese sentido se necesita una "segunda ola democratizadora" pero que altere radicalmente tanto el esquema de prioridades en la asignación de los recursos públicos, permitiendo la implementación de políticas compensatorias con discriminación positiva, como las prácticas institucionales y pedagógicas vigentes. Dada la fuerte segmentación institucional es evidente que tales políticas serán más efectivas si se operacionalizan en base a una rigurosa selección de unidades educativas. La ausencia de políticas activas en ese nivel educativo no sólo retardará su universalización, sino que al alcanzarla, el perfil de la inequidad educativa se habrá agravado aún más.

\section{Bibliografia}

Aitking, M. y Longford, N. (1986). Statistical modelling issues in school effectiveness. Journal of the Royal Statistical Society A, 149, pp.1-42.

Alexander, L. and Simmons, J. (1975). The determinants of school achievement in developing countries: the educational production function. World Bank Staff Working Paper, $N^{\circ}$ 201. Washington: World Bank.

Bourdieu, P. (2000). Las formas del capital. Trad. de Andrés García Inda en: Poder, Derecho y Clases Sociales, Cap. IV. Bilbao:Desclée de Brouwer.

Bowles, S. y Levin, H. (1968). The determinants of scholastic achievement. An appraisal of some recent evidence. Journal of Human Resources, invierno, vol. 3 (1), pp. 3-24.

Bryk, A. y Raudenbush, S. (1992). Hierarchical Linear Models for Social and Behavioral Research: Applications and Data Analysis Methods. Newbury Park, CA:Sage.

Cervini, R (1999). Calidad y Equidad en la Educación Básica de Argentina. Factores Asociados al Logro Escolar, $\mathrm{N}^{\mathrm{o}}$ 5. Buenos Aires: Ministerio de Cultura y Educación de la Nación.

Cervini, R (2002a) La distribución social de los rendimientos escolares. En: El rendimiento escolar en Argentina - Análisis de resultados y factores. Emilio Tenti (Org.), Buenos Aires: Editorial Losada.

Cervini, R (2002b). Desigualdades en el Logro Académico y Reproducción Cultural en Argentina - Un modelo de tres niveles. Revista Mexicana de Investigación Educativa, Vol.VIII (16). México: Consejo Mexicano de Investigación Educativa (en prensa).

Coleman, J. (1969) The concept of Equality of Educational Opportunity. Harvard Educational Review. Special Issue 'Equal Educational Opportunity'.

Coleman, J. (1975) What is meant by 'an equal educational opportunity'. Oxford Review of Education, Vol. 1 (1), pp.27-29.

Coleman, J., Campbell, E., Hobson C., McPartland, J., Mood, A., Weinfeld, F. y York, R. (1966). Equality of Educational Opportunity, U.S. Department of Health, Education and Welfare, Office of Education. Washington: Government Printing Office.

Cuttance, P. (1987). Modelling variation in the effectiveness of schooling. Edinburgh: Centre for Educational Sociology.

Fraser,B., Walberg, H, Welch, W. y Hattie,J. (1987). Syntheses of educational productivity research. International Journal of Educational Research, vol. 11 (2), pp.245-252. 
Goldstein, H. (1987) Multilevel Models in Educational and Social Research. Griffin. London

Guttman, A. (2001). La educación democrática Una teoría política de la educación. Barcelona: Editorial Paidós.

Heyneman, S. (1976). Influences on Academic Achievement: A Comparison of Results from Uganda and More Industrialized Societies. Sociology of Education, 49, pp. 200-11.

Heyneman, S. (1980). Difference between Developed and Developing Countries: Comments on Simmons and Alexander's "Determinants of School Achievement". Economic Development and Cultural Change, 28 (2), pp. 403-6.

Heyneman, S. y Loxley W. (1982). Influence on Academic Achievement across High and Low Income Countries: A Reanalysis of IEA Data. Sociology of Education, 55 (1), pp. 13-21.

Jencks,C. et.al. (1972). Inequality: a reassessment of the effects of family and schooling in America. New York: Basic.

Lareau,A. (1987). Social class diferences in family-school relationships: The importance of cultural capital. Sociology of Education, vol.60 (2), pp. 73-85.

Lockheed, M., Fuller, B. y Nyirondo, R. (1989). Family effects on students' achievement in Thailand and Malawi. Sociology of Education, vol. 62 (October), pp. 239-256.

Munoz Izquierdo, C. y Ulloa, M. (1992). Cuatro tesis sobre el origen de las desigualdades educativas - Una reflexión apoyada en el caso de México. Revista Latinoamericana de Estudios Educativos, vol. XXII (2), pp. 11-58.

Ocampo, J. (2000). Equidad, Desarrollo y Ciudadanía. CEPAL Documento Central 20002001. Santiago: CEPAL.

Rawls, J. (1979) Teoría de la Justicia. Madrid: Fondo de Cultura Económica

Rawls, J. (1982). Social Unity and Primary Goods. En: A. Sen y B. Williams (Ed.)
Utilitarianism and Beyond, Cambridge: Cambridge University Press, pp.159-186.

Ridell, A. (1989). An alternative approach to the study of school effectiveness in Third World Countries. Comparative Education Review, Vol.33 (4), pp. 481-97.

Ridell, A. (1997). Assessing designs school effectiveness research and school improvement in developing countries. Comparative Education Review, vol.41 (2), pp. 178-204.

SIEMPRO (2001). No se trata de un ranking de calidad, se trata de un ranking de desigualdad y pobreza. Sistema de Información, Monitoreo y Evaluación de Programas Sociales SIEMPRO. Buenos Aires: SIEMPRO.

Schiefelbein, E. y Simmons, J. (1980) Los determinantes del rendimiento escolar. Reseña de la investigación para los países en desarrollo. Educación hoy, $\mathrm{n}^{\circ} 60$.

Van Parijs, P. (1992). ¿Qué es una sociedad justa? Buenos Aires: Ediciones Nueva Visión.

Walberg, H.J. (1986). Synthesis of research on teaching. En: M.C. Wittrock (Ed.). Handbook of research on teaching (pp.85-102). Washington: American Educational Research Association.

Wolff, L., Schiefelbein, E. y Valenzuela, J. (1993). Mejoramiento de la calidad de la educación primaria en América Latina y el Caribe: hacia el siglo XXI. Banco Mundial, América Latina y el Caribe, Programa de Estudios Regionales, Informe $\mathrm{N}^{\mathrm{o}} 28$.

\section{Notas}

[1] La Ley Federal de Educación de 1993 (Ley N $\mathrm{N}^{\circ}$ 24.195), extendió la educación básica obligatoria a 9 años $\mathrm{y}$ en consecuencia, los dos primeros años de la exSecundaria se tornaron obligatorios. Los tres o cuatro años superiores de la exSecundaria pasaron a formar el ciclo Polimodal que, según la ley, no es obligatorio. En este trabajo nos estamos refiriendo a la exSecundaria completa.

[2] Se trata del acceso a los siguientes bienes básicos: los poderes y prerrogativas adheridas a las funciones, los 
ingresos y las riquezas, y las bases sociales del respeto por sí mismo (prestigio y reconocimiento).

[3] Las libertades fundamentales, aunque bienes básicos, no son materia de distribución: todos los individuos deben tenerlas por igual. Ellas son la libertad política, de expresión, de reunión, de conciencia, de pensamiento, de propiedad y la protección contra la detención arbitraria y el secuestro.

[4] El fundamento último de este principio de no-exclusión es que los niños de hoy serán los adultos de mañana, quienes deberán deliberar y decidir entre "concepciones competitivas de buena vida y buenas sociedades" (Guttman,2001:68). Si alguna minoría o sector social es excluido no tendrá la capacidad deliberativa cuando adulto y por tanto, estará excluido de las deliberaciones cuyos resultados afectan su propia vida y la de sus descendientes.

[5] La forma "positiva" del principio constituye una generalización para cualquier variable bajo análisis y asegura la distribución de los aprendizajes de acuerdo al mérito ("a cada uno según su mérito o capacidad").

[6] Queda totalmente fuera de nuestro análisis la distribución de probabilidades de las posiciones ocupacionales, es decir, la relación entre origen social, credencial educativa y destino ocupacional.

[7] En el trabajo se observa que en 49 de los 80 estudios revisados que incluyeron el nivel socioeconómico de los padres, este factor mostró una relación positiva y significativa (Wolff, Schiefelbein y Valenzuela,1993: Cuadro 1-H).

[8] No se midió ningún aspecto del proceso de enseñanzaaprendizaje ("caja negra") y además, los insumos escolares relativos al docente no pudieron relacionarse con el grupo de alumnos, restando una posible mayor variabilidad y capacidad explicativa; la técnica de análisis de regresión utilizada ("step by step") no es apropiada puesto que, existiendo colinealidad entre las variables escolares y familiares, cuando éstas entran primero, no dejan varianza para explicar a los factores propiamente escolares (Bowles y Levin, 1968); considera la participación de las variables escolares y sociales aditivamente, sin tener en cuenta la probable interacción entre ellas.

[9] En el sistema educativo americano investigado por Coleman, no existían grandes diferencias entre las escuelas respecto a los insumos considerados (recursos didácticos, infraestructura, salarios de los docentes, etc.) y por lo tanto, era previsible su baja o ninguna eficacia o capacidad predictiva respecto de las diferencias de logros.
[10] De todas formas una revisión contemporánea a los trabajos de Heyneman (Schiefelbein y Simmons,1980) informaba que, de acuerdo a la mayoría de las investigaciones consideradas, en los países del Tercer Mundo la clase social ayuda significativamente a predecir el logro del estudiante y que las características familiares son más relevantes cuando se trata del aprendizaje en Lengua comparado con ciencias.

[11] Los argumentos, sin embargo, son extensible a otros resultados educativo y sus respectivos indicadores (escolarización, permanencia, repitencia, etc.).

[12] Para Bourdieu (2000), el capital cultural incluye tanto los diversos componentes de una determinada cultura (sistema simbólico) como también sus principales manifestaciones o productos. Existe en forma incorporada en el agente como resultado de la socialización, proceso de imposición y adquisición de arbitrarios culturales, realizada primariamente por la familia y de acuerdo a la clase social de pertenencia. Para el autor, la forma "objetivada" son tanto los libros, diccionarios, obras de arte, discursos, etc, como los aparatos, máquinas, herramientas, es decir, los productos, las "cosas".

[13] Está bien probado en la literatura no sólo la estrecha correlación entre repitencia y nivel socioeconómico, sino también su efecto "estigmatizante" negativo. Pero, dado que en el presente estudio, su efecto es "controlado" por todos los indicadores socioeconómicos, se asume que el efecto residual propio expresaría en gran parte, condiciones aptitudinales del alumno.

[14] Para el análisis, la provincia de Buenos Aires se divide en Gran Buenos Aires (Conurbano) y resto de la Provincia

[15] Estimaciones basadas en la Encuesta Permanente de Hogares (EPH) del Instituto Nacional de Estadística y Censos (INDEC), Mayo 1999.

[16] Esta estimación sitúa a Argentina en un punto intermedio entre la escolarización secundaria en América Latina y el Caribe, próxima al $60 \%$, y en los países desarrollados (100\%). Ver Cuadro 4-1 en Ocampo (2000).

\section{[17] Ver nota 12.}

[18] Algunas acciones de política social son realizadas involucrando al sistema educativo, pero por ello difícilmente pueden ser consideradas "educativas", aún cuando tengan efectos sobre los indicadores educacionales. Un ejemplo concreto en Argentina es la distribución de becas para jóvenes provenientes de hogares pobres que asisten al colegio, política que afecta el grado de incorporación y retención escolar. 
Cervini, Rubén (2002). Desigualdades Socioculturales en el Aprendizaje de Matemática y Lengua de la Educación Secundaria en Argentina. RELIEVE:, v.8, n. 2, p. 126-148.

http://www.uv.es/RELIEVE/v8n1/RELIEVEv8n2 1.htm

\section{ABOUT THE AUTHORS / SOBRE LOS AUTORES}

Rubén Alberto Cervini ( racervin@infovia.com.ar): Profesor Titular (por concurso) e Investigador en el Departamento de Ciencias Sociales de la Universidad Nacional de Quilmes, a cargo de los curso de Metodología de la Investigación Educativa y Evaluación de Programas y Sistemas Educativos de la carrera de Educación. Fue responsable del desarrollo y análisis de resultados de los instrumentos de recolección de información complementaria del Sistema Nacional de Evaluación de la Calidad Educativa, Ministerio de Educación y Cultura de la Nación. Además, ha realizado diversas consultorías con organismos nacionales e internacionales (OREALC/UNESCO, Banco Mundial) sobre esta misma temática. Su dirección postal es Salguero $24605^{\circ}$ Piso Capital Federal c.p. 2514 - Argentina

\section{ARTICLE RECORD / FICHA DEL ARTÍCULO}

\begin{tabular}{|c|c|}
\hline $\begin{array}{l}\text { Reference / } \\
\text { Referencia }\end{array}$ & $\begin{array}{l}\text { Cervini, R. (2002). Desigualdades Socioculturales en el Aprendizaje de Matemática y Lengua de la Educación } \\
\text { Secundaria en Argentina:Un modelo de tres niveles. Revista ELectrónica de Investigación y } \\
\text { EValuación Educativa, v. 8, n. 2. http://www.uv.es/RELIEVE/v8n1/RELIEVEv8n2_1.htm }\end{array}$ \\
\hline Title / Título & $\begin{array}{l}\text { Desigualdades Socioculturales en el Aprendizaje de Matemática y Lengua de la Educación } \\
\text { Secundaria en Argentina: un modelo de tres niveles (Sociocultural inequalities in the } \\
\text { learning of Language and Mathematics in Secondary Education in Argentina: A model of } \\
\text { three levels). }\end{array}$ \\
\hline Authors / Autores & Rubén Cervini \\
\hline $\begin{array}{l}\text { Review / } \\
\text { Revista }\end{array}$ & Revista ELectrónica de Investigación y EValuación Educativa (RELIEVE), v. 8, n. 2 \\
\hline ISSN & $1134-4032$ \\
\hline $\begin{array}{l}\text { Publication date / } \\
\text { Fecha de } \\
\text { publicación }\end{array}$ & 2002 (Reception Date: 22 May 2002; Publication Date: 5 September 2002) \\
\hline $\begin{array}{l}\text { Abstract / } \\
\text { Resumen }\end{array}$ & $\begin{array}{l}\text { Se analizan los efectos del origen social del alumno y del contexto socioeconómico de la } \\
\text { escuela y de la provincia, sobre el logro en Matemática y Lengua de alumnos del último año } \\
\text { de la educación secundaria en Argentina. Los datos analizados corresponden a más de } 150 \\
\text { mil alumnos en } 3.300 \text { colegios. Para el análisis se utilizaron modelos lineales de niveles } \\
\text { múltiples con tres niveles (alumno, escuela y Provincia). Los resultados se discuten a la luz } \\
\text { de la teoría de la justicia distributiva en educación y del concepto de igualdad de } \\
\text { oportunidad educativa. Se encontró que la distribución del rendimiento está afectado por la } \\
\text { extrema segmentación socioeconómica institucional del sistema educativo y que el capital } \\
\text { cultural familiar y contextual es el factor más importante. } \\
\text { This article analyzes the effects of family background and the socioeconomic context of the } \\
\text { school and state on students' achievements in mathematics and language at the end of } \\
\text { secondary education in Argentina. The study examined data of } 150,000 \text { students in } 3,300 \text { high } \\
\text { schools. Multilevel linear modeling with three levels (student, school and state) was used to } \\
\text { analyze the family background and context effects on achievement. The results are discussed } \\
\text { in term of the theory of distributive justice (Rawls) and the concept of equality of educational } \\
\text { opportunity (Coleman). It was found that the educational achievement is affected by the } \\
\text { strong socioeconomic segmentation of the institutional educational system and that the } \\
\text { cultural capital indicators are the most important predictors. }\end{array}$ \\
\hline $\begin{array}{l}\text { Keywords } \\
\text { Descriptores }\end{array}$ & $\begin{array}{l}\text { logro escolar, educación secundaria, contexto socioeconómico, antecedentes familiares, } \\
\text { capital cultural, segmentación educativa, matemáticas, lengua, desigualdad de } \\
\text { oportunidad educativa, Argentina. } \\
\text { educational achievement, high school, socioeconomic context, family background, social } \\
\text { origins, cultural capital, educational segmentation, Mathematics, Language, equality of } \\
\text { educational opportunity, Argentina }\end{array}$ \\
\hline
\end{tabular}


Cervini, Rubén (2002). Desigualdades Socioculturales en el Aprendizaje de Matemática y Lengua de la Educación Secundaria en Argentina. RELIEVE:, v.8, n. 2, p. 126-148.

http://www.uv.es/RELIEVE/v8n1/RELIEVEv8n2 1.htm

\begin{tabular}{|l|l|}
\hline $\begin{array}{l}\text { Institution / } \\
\text { Institución }\end{array}$ & Universidad Nacional de Quilmes, Argentina \\
\hline $\begin{array}{l}\text { Publication site / } \\
\text { Dirección }\end{array}$ & $\underline{\text { http://www.uv.es/RELIEVE }}$ \\
\hline Language / Idioma & Español (Title, abstract and keywords in english) \\
\hline
\end{tabular}

\section{Revista ELectrónica de Investigación y EValuación Educativa (RELIEVE)}

[ ISSN: 1134-4032 ]

(C) Copyright 2002, RELIEVE. Reproduction and distribution of this articles it is authorized if the content is no modified and their origin is indicated (RELIEVE Journal, volume, number and electronic address of the document). // C Copyright 2002, RELIEVE. Se autoriza la reproducción y distribución de este artículo siempre que no se modifique el contenido y se indique su origen (RELIEVE, volumen, número y dirección electrónica del documento). 